\title{
$p$-Dominance and Equilibrium Selection under Perfect Foresight Dynamics
}

\author{
Daisuke Oyama* \\ Graduate School of Economics, University of Tokyo \\ Hongo, Bunkyo-ku, Tokyo 113-0033, Japan \\ doyama@grad.e.u-tokyo.ac.jp
}

January 12, 2000; revised October 5, 2001

\begin{abstract}
This paper studies equilibrium selection based on a class of perfect foresight dynamics and relates it to the notion of $p$-dominance. A continuum of rational players are repeatedly and randomly matched to play a symmetric $n \times n$ game. There are frictions: opportunities to revise actions follow independent Poisson processes. The dynamics has stationary states, each of which corresponds to a Nash equilibrium of the static game. A strict Nash equilibrium is linearly stable under the perfect foresight dynamics if, independently of the current action distribution, there exists a consistent belief that any player necessarily plays the Nash equilibrium action at every revision opportunity. It is shown that a strict Nash equilibrium is linearly stable under the perfect foresight dynamics with a small degree of friction if and only if it is the $p$-dominant equilibrium with $p<1 / 2$. It is also shown that if a strict Nash equilibrium is the $p$-dominant equilibrium with $p<1 / 2$, then it is uniquely absorbing (and globally accessible) for a small friction (but not vice versa). Set-valued stability concepts are introduced and their existence is shown. Journal of Economic Literature Classification Numbers: C72, C73.

Key Words: equilibrium selection; perfect foresight dynamics; linear stability; $p$-dominance.
\end{abstract}

\footnotetext{
*I would like to thank Akihiko Matsui for his advice and guidance. I am also grateful to Josef Hofbauer, Kazuya Kamiya, Toshimasa Maruta, Tomoyuki Takenawa, an associate editor, and especially an anonymous referee for helpful comments.
} 


\section{Introduction}

The concept of Nash equilibrium (Nash [18]) has played a central role in the field of game theory and economics. However, in games with multiple strict Nash equilibria such as coordination games, it is difficult to predict the outcomes, which limits the predictive power of the theory and gives a motivation for the study of equilibrium selection. Recent approaches have addressed this issue by embedding the normal form game in the context of explicit dynamics to examine the stability of strict Nash equilibria under the dynamics. Well known are stochastic evolutionary dynamic models due to Kandori, Mailath, and Rob [11] and Young [20], in which boundedly rational players repeatedly play a normal form game. Those models can select a particular strict Nash equilibrium as a stochastically stable outcome in that it is played most frequently in the long run independently of the initial state.

In the present paper, we study the perfect foresight dynamics with Poisson revision opportunities, originally proposed by Matsui and Matsuyma [16] for $2 \times 2$ games. Our model has a single large population of identical rational players who are repeatedly and randomly matched to play a symmetric $n \times n$ game. Unlike the standard evolutionary approaches, players maximize their expected discounted payoffs. Each player must make a commitment to a particular action for a random time interval. Opportunities to revise actions follow Poisson processes, which are independent across players. By this assumption of friction, within a small time interval only a small fraction of players can change their actions, and hence a change in the action distribution is made in a continuous way. The stationary states of this dynamics correspond to the Nash equilibria of the static game. Our goal is to demonstrate that different strict Nash equilibria may have different stability properties.

The stability concept we employ is called linear stability. In this dynamics, if every player chooses action $a^{*}$ whenever given a revision opportunity, then the resulting path of action distribution converges linearly to the state where all players play $a^{*}$. A Nash equilibrium is said to be linearly stable under the perfect foresight dynamics if for any initial state, the linear path that converges to the corresponding stationary state is a perfect foresight equilibrium path. ${ }^{1}$ That is, the linearly stable equilibrium $\left(a^{*}, a^{*}\right)$ is such that, whatever the current action distribution is, if all players share a common belief that any player, given an opportunity, necessarily plays action $a^{*}$, then they actually play action $a^{*}$ at every opportunity. In other words, if players agree on a certain action and have no incentive to deviate from the agreement regardless of the actions to which they are currently committing, then this action constitutes a linearly stable equilibrium.

Our main result is that the linearly stable equilibrium is characterized

\footnotetext{
${ }^{1} \mathrm{~A}$ perfect foresight equilibrium path need not be a straight line in general.
} 
by the notion of $p$-dominance (Morris, Rob, and Shin [17], Kajii and Morris [10]). An action pair in a two-player game is said to be a $p$-dominant equilibrium if each action is a best response to any belief that the other player takes the action in this pair with probability at least $p$. It is shown that a (pure strategy) Nash equilibrium is linearly stable under the perfect foresight dynamics with a sufficiently small friction if and only if it is the $p$-dominant equilibrium with $p<1 / 2$.

The notion of $p$-dominance was introduced for an equilibrium selection criterion based on the incomplete information approach. Morris, Rob, and Shin [17] and Kajii and Morris [10] show that the $p$-dominant equilibrium with $p<1 / 2$ is selected in the sense that it is robust to incomplete information, i.e., behavior close to it is played in an equilibrium of every nearby incomplete information game.

We then examine alternative stability concepts introduced by Matsui and Matsuyma [16]. A Nash equilibrium state $x^{*}$ is absorbing if for every initial state close enough to $x^{*}$, any equilibrium path must converge to $x^{*} ; x^{*}$ is globally accessible if for any initial state, there exists an equilibrium path converging to $x^{*} .^{2}$ By definition, a linearly stable state is globally accessible. The concept of absorption incorporates local stability, while globally accessibility and linear stability concern global stability. We show that a strict Nash equilibrium that is linearly stable is also absorbing (and globally accessible). Therefore, for strict Nash equilibria, a linearly stable state in fact has a local stability property.

This, together with our main theorem stated above, implies that if a strict Nash equilibrium is the $p$-dominant equilibrium with $p<1 / 2$, then it is uniquely absorbing and globally accessible when the friction is sufficiently small. $^{3}$ Since for symmetric $2 \times 2$ games, $p$-dominance with $p<1 / 2$ is reduced to risk-dominance à la Harsanyi and Selten [7], this result extends that of Matsui and Matsuyama [16], who show that for $2 \times 2$ games the unique absorbing and globally accessible state for a small friction corresponds to the risk-dominant equilibrium. ${ }^{4}$

The $p$-dominant equilibrium with $p<1 / 2$ is also selected by other evolutionary models. Kandori and Rob [12], Maruta [14], and Ellison [4] show that the $p$-dominant equilibrium with $p<1 / 2$ is uniquely selected by equilibrium selection criteria based on stochastic evolutionary dynamics, i.e., it is the long run equilibrium (Kandori and Rob [12]), the stochastically

\footnotetext{
${ }^{2}$ There may exist multiple equilibrium paths due to the assumption of perfect foresight. Therefore, it is possible that a state is globally accessible but not absorbing.

${ }^{3}$ Hofbauer and Sorger [9] independently obtain a similar result for asymmetric $N$-player games with linear incentives.

${ }^{4}$ Similar selection results for $2 \times 2$ games are obtained by other approaches. Those are the payoff-uncertainty approach of Carlsson and van Damme [3], and the evolutionary approach of Kandori, Mailath, and Rob [11] and Young [20]. For two-action games with more than three players, however, the coincidence may break (Kim [13]).
} 
stable equilibrium (Maruta [14]), or the long run stochastically equilibrium (Ellison [4]). Our results supply another justification for $p$-dominance as a selection tool that differs from those by the incomplete information approach and by the stochastic evolutionary approach.

There are games which do not possess the $p$-dominant equilibrium with $p<1 / 2$. We provide an example in which a $p$-dominant equilibrium with $p \geq 1 / 2$ is absorbing and globally accessible when the friction is small. This shows that a strict Nash equilibrium that is absorbing and globally accessible for a small friction is not necessarily $p$-dominant for $p<1 / 2 .^{5}$

There also exist games in which no state is stable. In order to determine which equilibria are likely selected, it is natural to ask if a set of states is stable in some sense. Following Gilboa and Matsui [6], we introduce a setvalued stability concept called the cyclically stable set. We also propose an alternative concept called the globally accessible set. It is shown that at least one cyclically stable set always exists, and that the union of all cyclically stable sets is the globally accessible set, which implies the existence of the globally accessible set.

The perfect foresight dynamics with Poisson revision opportunities is also studied by Hofbauer and Sorger [8], who apply the dynamics to potential games. They consider the global maximization of the potential function which is applicable to potential games, and show that for the class of potential games the unique maximizer of the potential function is the absorbing and globally accessible state for a small friction. Their analysis is restricted to the class of symmetric two-player games with a symmetric payoff matrix and of those linearly equivalent to them. ${ }^{6}$

The selection results based on the perfect foresight approach are so far in agreement with those by the incomplete information approach. The $p$ dominant equilibrium with $p<1 / 2$ is robust to incomplete information (Morris, Rob, and Shin [17], Kajii and Morris [10]). For the class of potential games, Ui [19] shows that a Nash equilibrium that maximizes the potential function is robust to incomplete information. Based on the payoffuncertainty approach due to Carlsson and van Damme [3], Frankel, Morris, and Pauzner [5] obtain the noise-independent selection of the $p$-dominant equilibrium with $p<1 / 2$ and the potential maximizer.

Another related work is conducted by Burdzy, Frankel, and Pauzner [2]. They apply techniques from the payoff-uncertainty approach to dynamic games with payoff shocks in a similar environment to ours. In their model, a continuum of players are repeatedly and randomly matched to play a $2 \times 2$ coordination game, with frictions in action revisions, but whose payoff ma-

\footnotetext{
${ }^{5}$ The example also shows that our equilibrium selection approach based on the perfect foresight dynamics with frictions and those based on stochastic evolutionary dynamics may lead to different selections in general.

${ }^{6}$ Hofbauer and Sorger [9] extend the result to $N$-player games with potential functions, where every player has the same payoff function.
} 
trix changes randomly over time. They show that iterated conditional dominance leads to the selection of the risk-dominant equilibrium through a contagion argument similar to that of Carlsson and van Damme [3]. When the friction is sufficiently small, every player necessarily plays the risk-dominant action at the revision opportunity. It remains open whether their approach yields the same selection as that by the present paper beyond the class of $2 \times 2$ games.

The organization of this paper is as follows. Section 2 sets up our model of perfect foresight dynamics, proves the existence theorem for equilibrium paths, and introduces the concept of linear stability. Section 3 reviews the notion of $p$-dominance of strict Nash equilibria. Section 4 establishes the main result, which states the equivalence between linear stability and $p$-dominance with $p<1 / 2$. Section 5 examines the stability concepts introduced by Matsui and Matsuyama [16]. Section 6 provides some examples. Section 7 discusses set-valued stability concepts. Section 8 concludes.

\section{Perfect Foresight Dynamics}

In this section we set up the model of perfect foresight dynamics, establish the existence theorem for equilibrium paths, and introduce a stability concept. We denote by $\mathbb{R}^{n}$ the $n$-dimensional real space with the sup norm $|\cdot|$, by $\Delta^{n-1}$ the $(n-1)$-dimensional simplex, and by $e_{i}$ its $i$ th vertex. For $x \in \Delta^{n-1}$ and $\varepsilon>0, B_{\varepsilon}(x)$ denotes the $\varepsilon$-neighborhood of $x$ relative to $\Delta^{n-1}$, i.e., $B_{\varepsilon}(x)=\left\{y \in \Delta^{n-1}|| y-x \mid<\varepsilon\right\}$.

\section{$2.1 \quad$ Model}

We consider a symmetric two-player game with $n \geq 2$ actions. The action set of the game is denoted by $A=\left\{a_{1}, \ldots, a_{n}\right\}$, and the payoff matrix is $\left(u_{i j}\right)$, where $u_{i j}$ is the payoff received by a player using action $a_{i}$ against an opponent playing action $a_{j}$. In this paper, we shall only consider symmetric Nash equilibria. This game is played repeatedly in a society with a continuum of identical, anonymous players. At each point in time, players are matched randomly to form pairs and play the game anonymously. We assume that players cannot switch actions at every point in time. Instead, every player must make a commitment to a particular action for a random time interval. Time instants at which each player can switch actions follow a Poisson process with the mean arrival rate $\lambda$. The processes are independent across players.

The action distribution in the society at time $t$ is denoted by

$$
x(t)=\left(x_{1}(t), \ldots, x_{n}(t)\right) \in \Delta^{n-1},
$$

where $x_{i}(t)$ is the fraction of players who are committing to action $a_{i}$ at time $t$. Due to the assumption that the switching times follow independent Pois- 
son processes with arrival rate $\lambda, x_{i}(\cdot)$ is Lipschitz continuous with Lipschitz constant $\lambda$, which implies in particular that it is differentiable at almost all $t \in[0, \infty)$. A rational player anticipates the future evolution of action distribution $x(t)$, and, if given the opportunity to switch actions, commits to the action that maximizes the expected discounted payoff. Since the duration of the commitment has an exponential distribution with the mean $1 / \lambda$, the expected discounted payoff of committing to action $a_{i}$ at time $t$ with a given anticipated path $x(\cdot)$ is represented by

$$
V_{i}(t)=(\lambda+\theta) \int_{0}^{\infty} e^{-(\lambda+\theta) s} \sum_{k=1}^{n} x_{k}(t+s) u_{i k} d s,
$$

where $\theta>0$ is a common discount rate.

Endowed with perfect foresight, players correctly anticipate the future evolution of $x(t)$. Hence, the action distribution path $x(\cdot)$ with an initial state $x^{0}$ satisfies the conditions

$$
\begin{aligned}
& \dot{x}_{i}(t) \in\left\{\begin{array}{lll}
\left\{\lambda\left(1-x_{i}(t)\right)\right\} & \text { if } V_{i}(t)>V_{j}(t) & \forall j \neq i \\
\left\{-\lambda x_{i}(t)\right\} & \text { if } V_{i}(t)<V_{j}(t) & \exists j \neq i \\
{\left[-\lambda x_{i}(t), \lambda\left(1-x_{i}(t)\right)\right]} & \text { otherwise, }
\end{array}\right. \\
& x(0)=x^{0} \in \Delta^{n-1}
\end{aligned}
$$

for all $t$ where $x_{i}(t)$ is differentiable. Given $x^{0} \in \Delta^{n-1}$, we call a Lipschitz continuous function $x(\cdot)$ such that equations (1)-(3) hold a perfect foresight equilibrium path from $x^{0}$. When, for instance, $V_{i}>V_{j}$ holds for all $j \neq i$, all players taking actions other than $a_{i}$ switch to $a_{i}$ at the revision opportunity. During the interval $[t, t+h)$, at most $\lambda h$ portion of players are given the opportunity to change their actions.

Finally, following Matsui and Matsuyama (1995), we define the degree of friction by $\delta=\theta / \lambda>0$.

\section{$2.2 \quad$ Existence}

The concept of the perfect foresight dynamics requires that players make rational choices based on their expectations about the future evolution of action distribution, and that those expectations coincide with the actual path of action distribution. We must make sure of the existence of such consistent expectations for any initial condition of action distribution. In order to prove the existence of perfect foresight equilibrium paths for games with more than two actions, we need a new technique which is based on a fixed point argument, while for $2 \times 2$ games studied in Matsui and Matsuyama [16], it can be established constructively. ${ }^{7}$

\footnotetext{
${ }^{7}$ Hofbauer and Sorger [9] also prove a similar existence result by a different method. They identify perfect foresight equilibrium paths with equilibrium paths of an associated differential game and prove the existence of the latter.
} 
Theorem 1 For each initial condition, there exists a perfect foresight equilibrium path.

Proof. See Appendix.

The basic idea of the proof is as follows. Fix an arbitrary initial condition $x^{0}$. Suppose that all players anticipate the evolution of the action distribution as, say, $x(\cdot)$ with $x(0)=x^{0}$, which is not necessarily consistent. If they played the game based on the belief of $x(\cdot)$, then the action distribution path, say, $y(\cdot)$ with $y(0)=x^{0}$ would evolve. We denote by $\Psi(x)$ the set of such $y(\cdot)$ 's. This correspondence $\Psi$ maps each path to the set of "best response paths" against it, and a fixed point (in a function space) of $\Psi$ is a perfect foresight equilibrium path. We shall apply Kakutani's fixed point theorem.

\subsection{Stability}

We state the following obvious but important fact for reference.

Proposition 0 A path $x(\cdot)$ such that $x(t)=x^{*}$ for all t is a perfect foresight equilibrium path if and only if $\left(x^{*}, x^{*}\right)$ is a Nash equilibrium of the static game.

Our analysis of equilibrium selection is to study the stability of the stationary states in the dynamics described by equations (1)-(3). We propose a stability concept called linear stability. We first define a linear path from $x^{\prime} \in \Delta^{n-1}$ to $x \in \Delta^{n-1}$ by

$$
x(t)=x-\left(x-x^{\prime}\right) e^{-\lambda t} .
$$

The action distribution moves along the linear path to the state where all players play action $a^{*}$ if every player chooses $a^{*}$ at revision opportunities.

Definition $1 x \in \Delta^{n-1}$ is linearly stable if for any $x^{\prime} \in \Delta^{n-1}$, the linear path from $x^{\prime}$ to $x$ is a perfect foresight equilibrium path from $x^{\prime}$. A Nash equilibrium of the static game is linearly stable under the perfect foresight dynamics if the corresponding state is linearly stable.

By definition, a linearly stable equilibrium $\left(a^{*}, a^{*}\right)$ is such that, whatever the current action distribution is, if all players share a common belief that any player, given an opportunity, necessarily chooses action $a^{*}$, then they actually choose action $a^{*}$ at every opportunity.

\section{$3 \quad p$-Dominance}

Let $G$ be a symmetric two-player normal form game with the action set $A=\left\{a_{1}, \ldots, a_{n}\right\}$ and the payoff matrix $\left(u_{i j}\right)$. Denote by $\Delta(A)$ the set of 
probability distributions on $A$. We define the notion of $p$-dominance for a symmetric Nash equilibrium of $G$ as follows.

Definition 2 (Morris, Rob, and Shin [17], Kajii and Morris [10]) Action profile $\left(a_{i}, a_{i}\right)$ is a $p$-dominant equilibrium of $G$ if for all $j=1, \ldots, n$, and all $\pi \in \Delta(A)$ with $\pi\left(a_{i}\right) \geq p$,

$$
\sum_{k=1}^{n} \pi\left(a_{k}\right) u_{i k} \geq \sum_{k=1}^{n} \pi\left(a_{k}\right) u_{j k} .
$$

That is, playing $a_{i}$ is a best response if the player believes that the other player will play $a_{i}$ with probability at least $p$. Note that $\left(a_{i}, a_{i}\right)$ is a Nash equilibrium if and only if $\left(a_{i}, a_{i}\right)$ is a $p$-dominant equilibrium for some $p$. For a strict Nash equilibrium, this definition is equivalent to the following.

Definition 3 Action profile $\left(a_{i}, a_{i}\right)$ is a strict $p$-dominant equilibrium of $G$ if for all $j \neq i$, and all $\pi \in \Delta(A)$ with $\pi\left(a_{i}\right)>p$,

$$
\sum_{k=1}^{n} \pi\left(a_{k}\right) u_{i k}>\sum_{k=1}^{n} \pi\left(a_{k}\right) u_{j k} .
$$

There exist nongeneric games where a $p$-dominant equilibrium is not a strict $p$-dominant equilibrium. For example, in a degenerate game where payoffs are constant for any pair of actions, every action profile is a 0dominant equilibrium while no action profile is a strict $p$-dominant equilibrium for any $p$. For a generic choice of payoffs, however, any $p$-dominant equilibrium is a strict $p$-dominant equilibrium.

Notice that if action pair $\left(a_{i}, a_{i}\right)$ is a $p$-dominant equilibrium for some $p$, then $\left(a_{i}, a_{i}\right)$ is a $q$-dominant equilibrium for any $q$ with $p \leq q \leq 1$. Therefore, we are interested in the smallest $p$ for which $\left(a_{i}, a_{i}\right)$ is a $p$-dominant equilibrium.

Morris, Rob, and Shin [17] and Kajii and Morris [10] show that the $p$ dominant equilibrium with $p<1 / 2$ is selected in the sense that it is robust to incomplete information. That is, behavior close to it is played in an equilibrium of every nearby incomplete information game.

The notion of $p$-dominance is a generalization of Harsanyi and Selten's [7] notion of risk-dominance. For symmetric $2 \times 2$ games, the $p$-dominant equilibrium with $p<1 / 2$ is equivalent to the risk-dominant equilibrium. Consider a symmetric $2 \times 2$ game with the payoff matrix

$$
\left(u_{i j}\right)=\left(\begin{array}{ll}
a & c \\
b & d
\end{array}\right)
$$

with $a>b$ and $c<d$. In this game, $\left(a_{1}, a_{1}\right)$ is a $\mu$-dominant equilibrium and $\left(a_{2}, a_{2}\right)$ is a $(1-\mu)$-dominant equilibrium, where $\mu=(d-c) /\{(a-b)+(d-c)\}$. $\left(a_{1}, a_{1}\right)$ risk-dominates $\left(a_{2}, a_{2}\right)$ if and only if $a-b>d-c$ or $\mu<1 / 2$.

Finally, we state the following claim, which one can easily show. 
Claim 1 Suppose that $\left(a_{i}, a_{i}\right)$ and $\left(a_{j}, a_{j}\right)$ are distinct strict Nash equilibria of $G$. Take the smallest $p, q$ such that $\left(a_{i}, a_{i}\right)$ and $\left(a_{j}, a_{j}\right)$ are $p$-dominant and $q$-dominant, respectively. Then $p+q \geq 1$. In particular, if $G$ is a two-action game, then $p+q=1$.

\section{Main Result}

This section presents the main theorem, which exhibits the equivalence between $p$-dominance with $p<1 / 2$ and linear stability under the perfect foresight dynamics with a small degree of friction.

Theorem 2 There exists $\bar{\delta}>0$ such that a symmetric pure strategy Nash equilibrium is linearly stable for all $\delta<\bar{\delta}$ if and only if it is the $p$-dominant equilibrium with $p<1 / 2$.

This theorem follows from the lemma below. Recall that the $i$ th vertex $e_{i}$ of the simplex $\Delta^{n-1}$ corresponds to action $a_{i}$.

Lemma 1 Suppose that $\left(a_{i}, a_{i}\right)$ is a p-dominant equilibrium. Then, $e_{i}$ is linearly stable if and only if $p \leq 1 /(2+\delta)$.

Proof. First, we show that if $p \leq 1 /(2+\delta)$, then for any initial condition $x^{0}$, the linear path to $e_{i}$ satisfies the equilibrium condition, that is, for all $j \neq i, V_{i}(t) \geq V_{j}(t)$ for all $t$ along this linear path.

The expected discounted payoff to action $a_{j}$ at $t$ along the linear path from $x^{0}$ to $e_{i}$ is given by

$$
\begin{aligned}
& V_{j}(t)=(\lambda+\theta) \int_{0}^{\infty} e^{-(\lambda+\theta) s}\left[\left\{1-\left(1-x_{i}^{0}\right) e^{-\lambda(t+s)}\right\} u_{j i}\right. \\
&\left.+\sum_{k \neq i} x_{k}^{0} e^{-\lambda(t+s)} u_{j k}\right] d s \\
&= \sum_{k=1}^{n} \pi\left(a_{k}\right) u_{j k},
\end{aligned}
$$

where $\pi \in \Delta(A)$ is such that

$$
\pi\left(a_{i}\right)=1-\left(1-x_{i}^{0}\right) e^{-\lambda t} \frac{1+\delta}{2+\delta}, \text { and } \pi\left(a_{j}\right)=x_{j}^{0} e^{-\lambda t} \frac{1+\delta}{2+\delta} \text { for } j \neq i .
$$

Here,

$$
\begin{gathered}
\pi\left(a_{i}\right) \geq \frac{1}{2+\delta}+x_{i}^{0} \frac{1+\delta}{2+\delta} \\
\geq \frac{1}{2+\delta} \geq p
\end{gathered}
$$


since $e^{-\lambda t} \leq 1$ and $0 \leq x_{i}^{0} \leq 1$. It follows that $V_{i}(t) \geq V_{j}(t)$ for all $j$ from the supposition that $\left(a_{i}, a_{i}\right)$ is a $p$-dominant equilibrium.

To show the converse, suppose that $p>1 /(2+\delta)$. There then exists a probability distribution $\pi \in \Delta(A)$ with $\pi\left(a_{i}\right)=1 /(2+\delta)$ such that

$$
\sum_{k=1}^{n} \pi\left(a_{k}\right) u_{i k}<\sum_{k=1}^{n} \pi\left(a_{k}\right) u_{j k}
$$

for some $j \neq i$. It follows that for an initial condition $x^{0}$ with $x_{i}^{0}=0$ and $x_{k}^{0}=\pi\left(a_{k}\right)(2+\delta) /(1+\delta)$, the linear path from $x^{0}$ to $e_{i}$ does not satisfy the equilibrium condition, since

$$
V_{i}(0)-V_{j}(0)=\sum_{k=1}^{n} \pi\left(a_{k}\right) u_{i k}-\sum_{k=1}^{n} \pi\left(a_{k}\right) u_{j k}<0
$$

holds along this path. This implies that $e_{i}$ is not linearly stable.

Example 4.1 We apply our main result to the class of marginal bandwagon games (Kandori and Rob [12]), which includes pure coordination games. Let us consider a symmetric two-player game $G$ with the action set $A=$ $\left\{a_{1}, \ldots, a_{n}\right\}$, and denote the payoff matrix by $\left(u_{i j}\right) . G$ is said to have the marginal bandwagon property (MBP) if $u_{i i}-u_{j i}>u_{i k}-u_{j k}$ for all distinct $i, j, k$. Action profile $\left(a_{i}, a_{i}\right)$ is said to be a pairwise risk-dominant equilibrium if it is risk-dominant in the reduced game $\left\{a_{i}, a_{i}\right\} \times\left\{a_{j}, a_{j}\right\}$ for every $j \neq i$.

Suppose that $G$ has MBP and has the pairwise risk-dominant equilibrium $\left(a_{i}, a_{i}\right)$. Then $\left(a_{i}, a_{i}\right)$ is the $p$-dominant equilibrium with $p<1 / 2$, as shown in Kandori and Rob [12]. Thus, it is linearly stable for a small friction by Theorem 2. In particular, we consider a pure coordination game, whose payoff matrix $\left(u_{i j}\right)$ is such that $u_{11}>u_{22}>\cdots>u_{n n}>0$ and $u_{i j}=0$ for $i \neq j$. A Pareto dominant equilibrium $\left(a_{1}, a_{1}\right)$, which is pairwise riskdominant and therefore $p$-dominant for $p<1 / 2$, is linearly stable for a small friction.

\section{Absorption and Global Accessibility}

This section examines alternative stability concepts introduced by Matsui and Matsuyama [16].

Definition 4 (Matsui and Matsuyama [16]) (a) $x \in \Delta^{n-1}$ is accessible from $x^{\prime} \in \Delta^{n-1}$, if there exists a perfect foresight equilibrium path from $x^{\prime}$ that converges to $x . x \in \Delta^{n-1}$ is globally accessible if it is accessible from any $x^{\prime} \in \Delta^{n-1} . x \in \Delta^{n-1}$ is locally accessible if there exists $\varepsilon>0$ such that $x$ is accessible from any $x^{\prime} \in B_{\varepsilon}(x)$. 
(b) $x \in \Delta^{n-1}$ is absorbing if there exists $\varepsilon>0$ such that any perfect foresight equilibrium path from $B_{\varepsilon}(x)$ converges to $x . x \in \Delta^{n-1}$ is fragile if it is not absorbing.

We say that a Nash equilibrium is globally accessible (absorbing, respectively) if the corresponding state is globally accessible (absorbing, respectively). By definition, linear stability implies global accessibility. It will be shown that a linearly stable state that corresponds to a strict Nash equilibrium is also absorbing.

The following lemma relates the stability concepts stated above to the notion of $p$-dominance. If the state $e_{i}$, where all players play action $a_{i}$, corresponds to a strict $p$-dominant equilibrium with a sufficiently small $p$, then in a neighborhood of $e_{i}$, every player chooses $a_{i}$ and thus any perfect foresight equilibrium path must converge to $e_{i}$.

Lemma 2 (a) Suppose that $\left(a_{i}, a_{i}\right)$ is a p-dominant equilibrium. Then, $e_{i}$ is globally accessible if $p \leq 1 /(2+\delta)$.

(b) Suppose that $\left(a_{i}, a_{i}\right)$ is a strict p-dominant equilibrium. Then, $e_{i}$ is absorbing if $p<(1+\delta) /(2+\delta)$.

Proof. (a) This immediately follows from Lemma 1.

(b) We show that if $p<(1+\delta) /(2+\delta)$, then the equilibrium path $x(\cdot)$ from any $x^{0}$ sufficiently close to $e_{i}$ is unique and converges to $e_{i}$. Note that any feasible path from $x^{0}$ satisfies $x_{j}(t) \geq x_{j}^{0} e^{-\lambda t}$.

The expected discounted payoff to action $a_{j}$ at time 0 along the path $x(\cdot)$ is given by

$$
V_{j}(0)=\sum_{k=1}^{n} \pi\left(a_{k}\right) u_{j k},
$$

where $\pi \in \Delta(A)$ is such that

$$
\pi\left(a_{k}\right)=(\lambda+\theta) \int_{0}^{\infty} e^{-(\lambda+\theta) s} x_{k}(s) d s .
$$

If $x^{0}$ is sufficiently close to $e_{i}$ so that $p(2+\delta) /(1+\delta)<x_{i}^{0}<1$, then

$$
\begin{aligned}
\pi\left(a_{i}\right) & \geq(\lambda+\theta) \int_{0}^{\infty} e^{-(\lambda+\theta) s} x_{i}^{0} e^{-\lambda s} d s \\
& =x_{i}^{0} \frac{1+\delta}{2+\delta}>p .
\end{aligned}
$$

It follows that $V_{i}(0)>V_{j}(0)$ for all $j \neq i$ from the supposition that $\left(a_{i}, a_{i}\right)$ is a strict $p$-dominant equilibrium. This implies that $x_{i}^{0} \leq x_{i}(t)<1$, and $V_{i}(t)>V_{j}(t)$ for all $t$. Thus $x_{i}(t)=1-\left(1-x_{i}^{0}\right) e^{-\lambda t}$, and $\lim _{t \rightarrow \infty} x(t)=e_{i}$. 
Combining Lemma 1 and Lemma 2, we obtain the following result on the relationship between stability concepts. Whereas linear stability concerns global stability, it in fact has a local stability property for strict Nash equilibria.

Proposition 1 If a symmetric strict Nash equilibrium is linearly stable, then it is also absorbing.

From Lemma 2, we obtain the following theorem, which extends the result of Matsui and Matsuyama [16]. ${ }^{8}$

Theorem 3 If a symmetric strict Nash equilibrium is the p-dominant equilibrium with $p<1 / 2$, then it is absorbing for all $\delta$ and there exists $\bar{\delta}>0$ such that it is globally accessible for all $\delta<\bar{\delta}$

Remark 1. Hofbauer and Sorger [8] study potential games under the same dynamic environment and show that the unique maximizer of the potential function is the absorbing and globally accessible state for a small friction. Their analysis is restricted to the class of games that is linearly equivalent to games with a symmetric payoff matrix. An example to which their analysis does not apply is the marginal bandwagon game described by

$$
\left(u_{i j}\right)=\left(\begin{array}{lll}
6 & 0 & 0 \\
5 & 7 & 5 \\
4 & 5 & 8
\end{array}\right),
$$

which is taken from Kandori and Rob [12]. This game is not a potential game, while it has a $p$-dominant equilibrium with $p<1 / 2$. Nash equilibrium $\left(a_{3}, a_{3}\right)$ is $2 / 5$-dominant and therefore is uniquely absorbing and globally accessible for a small friction by Theorem 3 .

As mentioned earlier, $p$-dominance is a generalization of risk-dominance. Indeed, $p$-dominance with $p<1 / 2$ is equivalent to risk-dominance for symmetric $2 \times 2$ games. Theorem 3 can be paraphrased by saying that a manyaction generalization of risk-dominance is a sufficient condition for a Nash equilibrium being absorbing and globally accessible. Although the sufficient condition gives a limited characterization in general, it is enough to give a full characterization of the stability for symmetric $2 \times 2$ coordination games as studied in Matsui and Matsuyama [16]. We obtain the following known result as a corollary to Lemma 2 .

Corollary 1 (Matsui and Matsuyama [16]) Consider the case of symmetric two-action games with two symmetric strict Nash equilibria. Suppose that $\left(a_{i}, a_{i}\right)$ is a p-dominant equilibrium.

(a) $e_{i}$ is globally accessible if and only if $p \leq 1 /(2+\delta)$,

(b) $e_{i}$ is absorbing if and only if $p<(1+\delta) /(2+\delta)$.

\footnotetext{
${ }^{8} \mathrm{~A}$ similar result for asymmetric $N$-player games with linear incentives is independently obtained by Hofbauer and Sorger [9].
} 
Proof. The "if" part is the $n=2$ case of Lemma 2. We prove the "only if" part. Suppose that $\left(a_{j}, a_{j}\right)$ is a $q$-dominant equilibrium for $j \neq i$. Notice that $p+q=1$ by Claim 1 .

(a) If $p>1 /(2+\delta)$, then $q<(1+\delta) /(2+\delta)$. Thus it follows from the "if" part of (b) that $e_{j}$ is absorbing, which implies that $e_{i}$ is not globally accessible.

(b) If $p \geq(1+\delta) /(2+\delta)$, then $q \leq 1 /(2+\delta)$. Therefore, it follows from the "if" part of (a) that $e_{j}$ is globally accessible, which implies that $e_{i}$ is not absorbing.

Consider a symmetric $2 \times 2$ game with the payoff matrix given by

$$
\left(u_{i j}\right)=\left(\begin{array}{ll}
a & c \\
b & d
\end{array}\right),
$$

where $a>b$ and $c<d$. Write $\mu=(d-c) /\{(a-b)+(d-c)\}$. If $\mu<1 / 2$, then $\left(a_{1}, a_{1}\right)$ is absorbing and globally accessible for a small friction, as $\left(a_{1}, a_{1}\right)$ is a $\mu$-dominant equilibrium. Conversely, if $\left(a_{1}, a_{1}\right)$ is uniquely absorbing and globally accessible for some friction, then $\mu<1 / 2$, due to Corollary 1 . Therefore, a Nash equilibrium is uniquely absorbing and globally accessible for a small friction if and only if it is the $\mu$-dominant equilibrium with $\mu<1 / 2$, equivalently, the risk-dominant equilibrium.

We state some other stability properties. Recall that a strict Nash equilibrium is $p$-dominant for some $p<1$.

Proposition 2 Every symmetric strict Nash equilibrium is locally accessible independently of the friction.

Proof. Suppose that $\left(a_{i}, a_{i}\right)$ is a $p$-dominant equilibrium with $p<1$. It is sufficient to show that the linear path from any initial condition $x^{0}$ to state $e_{i}$ satisfies the equilibrium condition. This can be verified in precisely the same way as in the proof of Lemma 1.

Proposition 3 Every symmetric strict Nash equilibrium is absorbing for a sufficiently large friction.

Proof. This immediately follows from Lemma 2 .

Proposition 3 states that, in the case of a large friction, the dynamics is similar to the best response dynamics studied in Gilboa and Matsui [6] and Matsui [15].

\section{Examples}

For games with three or more actions, there are games that do not possess the $p$-dominant equilibrium with $p<1 / 2$. This section lists some simple 
examples, which show that: (i) a strict Nash equilibrium that is absorbing and globally accessible need not be $p$-dominant for $p<1 / 2$; (ii) there may not exist absorbing states nor globally accessible states.

\subsection{Young's Example}

The following example shows that the converse of Theorem 3 is not necessarily true, except for the case of two-action games. A $p$-dominant equilibrium with $p \geq 1 / 2$ may be absorbing or globally accessible for a small friction. Consider the $3 \times 3$ game with the payoff matrix given by

$$
\left(u_{i j}\right)=\left(\begin{array}{lll}
6 & 0 & 0 \\
5 & 7 & 5 \\
0 & 5 & 8
\end{array}\right),
$$

which is taken from Young [20]. In this game, $\left(a_{1}, a_{1}\right)$ is $7 / 8$-dominant; $\left(a_{2}, a_{2}\right)$ is $3 / 5$-dominant; $\left(a_{3}, a_{3}\right)$ is $5 / 8$-dominant. It can be verified that $\left(a_{3}, a_{3}\right)$ is absorbing for any degree of friction and globally accessible for a small friction $\delta .^{9}$ Figure 1 is a geometric representation of this game. One can verify the global accessibility of $\left(a_{3}, a_{3}\right)$ for a small friction by direct computation along the paths described below. When the society starts from a state below the dotted line, there exists a perfect foresight equilibrium path linearly converging to vertex 3 , along which players always switch to action $a_{3}$ at their first opportunities. On the other hand, when the society starts from a state above the dotted line, the linear path to vertex 3 does not satisfy the equilibrium condition. Given the belief of such a path, players choose $a_{2}$ rather than $a_{3}$, since $V_{2}(0)>V_{3}(0)$ holds for that path. A perfect foresight equilibrium path from a state above the dotted line is the path depicted in Figure 1, along which the society first moves toward vertex 2 and then changes its direction toward vertex 3 at the dotted line.

To show that $\left(a_{3}, a_{3}\right)$ is absorbing, verify first by direct computation that unless the initial state is close to $e_{1}, V_{2}(0)>V_{1}(0)$ holds for any feasible path. Therefore, any perfect foresight equilibrium path $x(\cdot)$ must satisfy $x_{1}(t)=x_{1}^{0} e^{-\lambda t}$ when it starts from a state close to $e_{3}$. Then verify again by direct computation that if the initial condition is sufficiently close to $e_{3}$, $V_{3}(0)>V_{2}(0)$ holds for any feasible path $x(\cdot)$ with $x_{1}(t)=x_{1}^{0} e^{-\lambda t}$.

Note that $\left(a_{2}, a_{2}\right)$ is the long-run equilibrium (Kandori and Rob [12]) and the stochastically stable equilibrium (Young [20]), while it is neither absorbing nor globally accessible when the friction is small. This example suggests that our equilibrium selection approach based on the perfect foresight dynamics and those based on the stochastic evolutionary dynamics lead to different predictions in general.

\footnotetext{
${ }^{9}$ Notice that the level of $p$-dominance of $\left(a_{3}, a_{3}\right)$ is not the smallest among those of three strict Nash equilibria.
} 


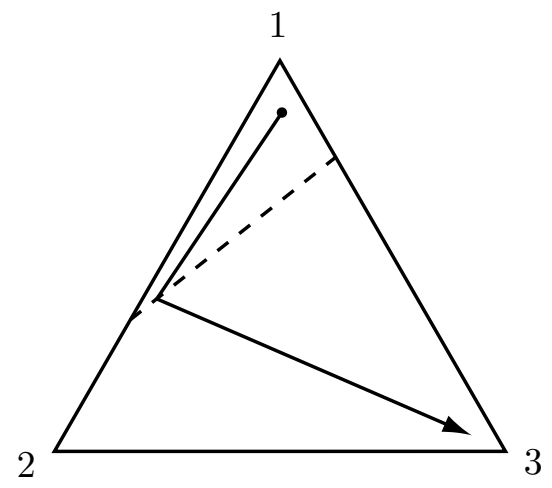

Figure 1

\subsection{Nonexistence}

In games that do not possess the $p$-dominant equilibrium with $p<1 / 2$, all states may be fragile for a small friction. Consider the following example: ${ }^{10}$

$$
\left(u_{i j}\right)=\left(\begin{array}{lll}
2 & 1 & 0 \\
0 & 2 & 1 \\
1 & 0 & 2
\end{array}\right) .
$$

Each strict Nash equilibrium is $2 / 3$-dominant. If $\delta>1$, then each strict equilibrium is absorbing due to Lemma 2 , while if $\delta \leq 1$, then each strict equilibrium is fragile since each linear path from $e_{i}$ to $e_{i-1}(\bmod 3)$ becomes a perfect foresight equilibrium path, which is checked by direct computation.

It is verified that each strict equilibrium is not globally accessible, either. We show that if $\delta \leq 1$, then $e_{1}$ is not accessible from $e_{3}$. Take any equilibrium path converging to $e_{1}$, and denote it $x(\cdot)$. There then exists $t$ such that

$$
x(s)=e_{1}-\left(e_{1}-x(t)\right) e^{-\lambda(s-t)}
$$

for all $s \geq t$. We denote by $T$ the infimum of such $t$ 's. We show that $x(\cdot)$ does not change its direction, i.e., $T=0$. Suppose that $T>0$. Then $V_{1}(T)=V_{i}(T)$ must hold for some $i \neq 1$, where $V_{i}(T)$ is the discounted expected payoff of choosing $a_{i}$ at $T$ along the path such that $x(t)=e_{1}-\left(e_{1}-\right.$ $x(T)) e^{-\lambda(t-T)}$ for $t \geq T$. One can verify that if $\delta \leq 1$, then $V_{1}(T)>V_{2}(T)$ for any linear path to $e_{1}$, unless $x(T)=e_{2}$. Therefore, $x(\cdot)$ does not move toward $e_{2}$ at $T-0$. Moreover, it can be verified that $V_{1}(T)=V_{3}(T)$ if and only if $x_{3}(T)=(2+\delta) / 3(1+\delta)$ and that $V_{1}(T)>V_{3}(T)$ if and only if

\footnotetext{
${ }^{10}$ Note that this example is generic.
} 
$x_{3}(T)<(2+\delta) / 3(1+\delta)$, which implies that $x(\cdot)$ does not move toward $e_{3}$ at $T-0$, either. Hence, we can conclude that $T=0$. One can verify that $V_{1}(0)<V_{3}(0)$ holds for the linear path from $e_{3}$ to $e_{1}$, thereby completing the proof.

\section{Set-Valued Stability Concepts}

As we see in Section 6.2, there may not exist absorbing states nor globally accessible states for a small friction. However, even in such cases there may be sets of states that are good asymptotic description of the long-run behavior. We introduce two set-valued stability concepts and show their existence.

We first redefine accessibility following Matsui [15]. Given a path $x$ : $[0, \infty) \rightarrow \Delta^{n-1}$, we say that $x^{*}$ is an accumulation point of $x(\cdot)$ if there exists a real sequence $\left\{t^{k}\right\}$ such that $t^{k} \rightarrow \infty$ and $x\left(t^{k}\right) \rightarrow x^{*}$ as $k \rightarrow \infty$. Note that any path $x(\cdot)$ has at least one accumulation point because of the compactness of $\Delta^{n-1}$.

Definition $5 x \in \Delta^{n-1}$ is directly accessible from $x^{\prime} \in \Delta^{n-1}$ if there exists a perfect foresight equilibrium path $x(\cdot)$ from $x^{\prime}$ such that $x$ is an accumulation point of $x(\cdot)$.

$x \in \Delta^{n-1}$ is accessible from $x^{\prime} \in \Delta^{n-1}$ if at least one of the following is satisfied: (i) $x$ is directly accessible from $x^{\prime}$; (ii) there exists a sequence $\left\{x^{k}\right\}$ converging to $x$ such that $x^{k}$ is accessible from $x^{\prime}$ for all $k$; (iii) $x$ is accessible from $y$ which is accessible from $x^{\prime}$.

Now we define a set-valued stability concept which is analogous to that of Gilboa and Matsui [6].

Definition 6 A nonempty subset $F^{*}$ of $\Delta^{n-1}$ is a cyclically stable set (with respect to the perfect foresight dynamics), or CSS, if

(a) no $x \notin F^{*}$ is accessible from any $x^{\prime} \in F^{*}$,

(b) every $x \in F^{*}$ is accessible from every $x^{\prime} \in F^{*}$.

An absorbing state, taken as a singleton, is a CSS.

We prove the existence of CSS. For each $x \in \Delta^{n-1}$, we define $R(x)$ to be

$$
R(x)=\left\{y \in \Delta^{n-1} \mid y \text { is accessible from } x\right\} .
$$

From the fact that every equilibrium path has an accumulation point, $R(x)$ is nonempty.

Now we state the existence theorem. The proof is by direct application of Gilboa and Matsui [6].

Theorem 4 Every game has at least one CSS. 
Proof. Observe that $R(x)$ is closed and $x^{\prime} \in R(x)$ implies $R\left(x^{\prime}\right) \subset R(x)$ by the definition of accessibility.

We consider the partially ordered set $\left(\{R(x)\}_{x \in \Delta^{n-1}}, \subset\right)$. Take any totally ordered subset of $\{R(x)\}_{x \in \Delta^{n-1}}$ and denote it by $\{R(x)\}_{x \in \Delta^{\prime}}$. Since for each $x \in \Delta^{\prime}, R(x)$ is a closed and nonempty subset of a compact set, $\bigcap_{x \in \Delta^{\prime}} R(x)$ is nonempty. Choose any $y \in \bigcap_{x \in \Delta^{\prime}} R(x)$. Since $R(y) \subset R(x)$ holds for all $x \in \Delta^{\prime}, R(y)$ is a lower bound of $\{R(x)\}_{x \in \Delta^{\prime}}$ in $\{R(x)\}_{x \in \Delta^{n-1}}$. Therefore, by Zorn's lemma, there exists a minimal element $R^{*}=R\left(x^{*}\right)$ in $\{R(x)\}_{x \in \Delta^{n-1}}$.

We claim that $R^{*}$ is a CSS. Indeed, for any $x \in R^{*}, R(x) \subset R^{*}$ holds, and since $R^{*}$ is a minimal set, $R(x)=R^{*}$ holds, which implies that no point outside $R^{*}$ is accessible from any point in $R^{*}$, and every point in $R^{*}$ is accessible form any point in $R^{*}$.

We introduce an alternative concept of stability.

Definition 7 A nonempty subset $G^{*}$ of $\Delta^{n-1}$ is a globally accessible set, or $G A S$, if $G^{*}$ is the minimum set among those satisfying the following:

(a) no $x \notin G^{*}$ is accessible from any $x^{\prime} \in G^{*}$,

(b) for all $x \in \Delta^{n-1}$, there exists $x^{\prime} \in G^{*}$ such that $x^{\prime}$ is accessible from $x$.

An absorbing and globally accessible state, taken as a singleton, is a GAS.

By definition, a GAS is unique if it exists. The following proposition ensures its existence.

Proposition 4 The union of all CSS's is the GAS.

Proof. Denote by $\mathcal{F}^{*}$ the family of all CSS's. By the definition of CSS, no $x \notin \cup \mathcal{F}^{*}$ is accessible from any $x^{\prime} \in \bigcup \mathcal{F}^{*}$. For any $x_{0} \in \Delta^{n-1}$, define $\mathcal{R}=\left\{R \in\{R(x)\}_{x \in \Delta^{n-1}} \mid R \subset R\left(x_{0}\right)\right\}$. By a similar argument to the proof of Theorem 4, $\mathcal{R}$ has a minimal element. Therefore, for each $x_{0} \in \Delta^{n-1}$, there exists a minimal element $R^{*}$ of $\{R(x)\}_{x \in \Delta^{n-1}}$ such that $R^{*} \subset R\left(x_{0}\right)$. Since $R^{*}$ is a CSS, there exists $x \in \cup \mathcal{F}^{*}$ such that $x$ is accessible from $x_{0}$. Obviously, $\bigcup \mathcal{F}^{*}$ is the minimum set, thereby completing the proof.

Theorem 5 Every game has the GAS.

Example 7.1 Consider the $4 \times 4$ game given by

$$
\left(u_{i j}\right)=\left(\begin{array}{llll}
2 & 1 & 0 & 0 \\
0 & 2 & 1 & 0 \\
1 & 0 & 2 & 0 \\
0 & 0 & 0 & 1
\end{array}\right) .
$$

The set $\left\{e_{1}, e_{2}, e_{3}\right\}$ is a CSS and a GAS. 


\section{Conclusion}

We have considered equilibrium selection for symmetric $n \times n$ games by embedding the original game into the perfect foresight dynamics with Poisson revision opportunities. Different strict Nash equilibria may have different stability properties under the dynamics, based on which we can select a particular equilibrium. We have established that a strict Nash equilibrium is linearly stable under the perfect foresight dynamics with a sufficiently small friction if and only if it is the $p$-dominant equilibrium with $p<1 / 2$. The result thus provides a dynamical characterization for the $p$-dominant equilibrium with $p<1 / 2$. We have also shown that the $p$-dominant equilibrium with $p<1 / 2$ is generically absorbing for any degree of friction.

\section{Appendix}

We introduce a Banach space $X$, the set of bounded functions $f:[0, \infty) \rightarrow$ $\mathbb{R}^{n}$ with norm

$$
\|f\|_{r}=\sup _{t \geq 0} e^{-r t}|f(t)|
$$

for $r>0$. Given any $x^{0} \in \Delta^{n-1}$, define $K \subset X$ to be

$$
K=\left\{x:[0, \infty) \rightarrow \Delta^{n-1} \mid x \text { is Lipschitz with constant } \lambda, \text { and } x(0)=x^{0}\right\} .
$$

Lemma $3 K$ is convex and compact.

Proof. Given an arbitrary $\varepsilon>0$, take $T$ which satisfies $e^{-r T}<\varepsilon$. Observe that $K$ is equi-continuous and uniformly bounded. By the Ascoli-Arzelà theorem, there exist finitely many elements $x^{1}, \ldots, x^{N} \in K$ such that for any $y \in K$,

$$
\sup _{t \in[0, T]}\left|y(t)-x^{k}(t)\right|<\varepsilon
$$

holds for some $k=1, \ldots, N$. Therefore, for any $y \in K$

$$
\left\|y-x^{k}\right\|_{r}<\varepsilon
$$

i.e., $K$ is totally bounded. Since $K$ is also complete, which is easily verified, $K$ is compact.

The fact that it is convex is easy to check.

Note that each $x \in K$ is differentiable almost everywhere, since it is Lipschitz continuous and hence of bounded variation. 
We rewrite the expected discounted payoff as the functional $V: K \times$ $[0, \infty) \rightarrow \mathbb{R}^{n}$ defined by

$$
V_{i}[x](t)=\int_{0}^{\infty} e^{-(\lambda+\theta) s} \sum_{k=1}^{n} x_{k}(t+s) u_{i k} d s,
$$

In order to establish the existence of equilibrium path, we consider the following functional differential inclusion which is equivalent to (1)-(3):

$$
\dot{x}(t) \in X[x](t, x(t)) \text { a.e., } \quad x(0)=x^{0} \in \Delta^{n-1},
$$

where

$$
X[x](t, x(t))=\left\{\lambda(\alpha-x(t)) \mid \alpha \in \Delta^{n-1}, \alpha_{i}=0 \text { if } i \notin M[x](t)\right\},
$$

and

$$
M[x](t)=\arg \max \left\{V_{i}[x](t) \mid i=1, \ldots, n\right\} .
$$

In equation (8), $\alpha \in \Delta^{n-1}$ is a convex combination of best response actions at $t$ against the belief of future path $x(\cdot)$.

A solution $x(\cdot) \in K$ to the functional differential inclusion (7) is a perfect foresight equilibrium path starting from the arbitrarily given initial condition $x^{0}$. Our goal is to prove that there exists a solution $x(\cdot) \in K$ to (7).

First, for a fixed $x \in K$, we consider the following differential inclusion:

$$
\dot{y}(t) \in X[x](t, y(t)) \text { a.e., } \quad y(0)=x^{0} \in \Delta^{n-1} .
$$

Lemma 4 There exists a solution $y(\cdot) \in K$ to (9).

Proof. The correspondence $X[x](\cdot, \cdot):[0, \infty) \times \Delta^{n-1} \rightarrow \mathbb{R}^{n}$ is convex- and compact-valued, and upper semi-continuous, since $V[x](\cdot)$ is continuous and therefore $M[x](\cdot)$ is upper semi-continuous. It follows that there exists a continuous function $y(\cdot)$, a solution to the problem (9) by the existence theorem for differential inclusion (see, e.g., Theorem 2.1.4 in Aubin and Cellina [1, p. 101]). Such $y(\cdot)$ is contained in $K$, since it satisfies (9).

We define the correspondence $\Psi: K \rightarrow K$ by

$$
\Psi(x)=\{y \in K \mid y(\cdot) \text { is a solution to }(9)\} .
$$

Notice that $y \in \Psi(x)$ if and only if

$$
\frac{1}{\lambda} \dot{y}(t)+y(t) \in F_{t}(x(\cdot)) \quad \text { a.e. }
$$

where the correspondence $F:[0, \infty) \times K \rightarrow \Delta^{n-1}$ is defined by

$$
F_{t}(x)=\left\{\alpha \in \Delta^{n-1} \mid \alpha_{i}=0 \text { if } i \notin M[x](t)\right\} .
$$

Note that $F_{t}(x)$ is compact and convex for any $t$ and $x$. 
Lemma $5 F_{t}(\cdot): K \rightarrow \Delta^{n-1}$ is upper semi-continuous.

Proof. For a fixed $t$, we define $v: \Delta^{n-1} \times K \rightarrow \mathbb{R}$ and $v^{*}: K \rightarrow \mathbb{R}$ by

$$
v(\alpha, x)=\sum_{i=1}^{n} \alpha_{i} V_{i}[x](t)
$$

and

$$
v^{*}(x)=\max \left\{V_{1}[x](t), \ldots, V_{n}[x](t)\right\} .
$$

Since $V[\cdot](t)$ is continuous, $v(\cdot, \cdot)$ and $v^{*}(\cdot)$ are continuous.

Let us show that $F_{t}(\cdot)$ has a closed graph. Let $\left\{x^{k}\right\}$ and $\left\{\alpha^{k}\right\}$ be such that $x^{k} \in K$ and $\alpha^{k} \in F_{t}\left(x^{k}\right)$ for all $k$, and assume $x^{k} \rightarrow x$ and $\alpha^{k} \rightarrow \alpha$. Notice that $\alpha^{k} \in F_{t}\left(x^{k}\right)$ if and only if $v\left(\alpha^{k}, x^{k}\right)=v^{*}\left(x^{k}\right)$. By the continuity of $v$ and $v^{*}$, we obtain $v(\alpha, x)=v^{*}(x)$, i.e., $\alpha \in F_{t}(x)$. Since the values are contained in a compact set $\Delta^{n-1}, F_{t}(\cdot)$ is upper semi-continuous.

Lemma 6 (a) $\Psi(x)$ is convex.

(b) $\Psi$ is compact-valued and upper semi-continuous.

In order to prove Lemma 6 , we need the following version of the convergence theorem (Theorem 1.4.1) due to Aubin and Cellina [1].

Convergence Theorem (Aubin and Cellina [1]) Let $X, Y$ be Banach spaces, $I$ an interval of $\mathbb{R}$, and $F: X \rightarrow Y$ a closed- and convex-valued upper semi-continuous correspondence. Suppose that $x^{k} \in X$ and $y^{k}: I \rightarrow Y$ satisfy $y^{k}(t) \in F\left(x^{k}\right)$ for almost all $t \in I$. If

1. $x^{k}$ converges strongly to $x$,

2. $y^{k}$ belongs to $L^{1}(I, Y)$ and converges weakly to $y$ in $L^{1}(I, Y)$,

then $y(t) \in F(x)$ for almost all $t \in I$.

Proof of Lemma 6. (a) Convexity is easy to check.

(b) We show that $\Psi$ has a closed graph. Let $\left\{x^{k}\right\}$ and $\left\{z^{k}\right\}$ be such that $x^{k} \in K$ and $z^{k} \in \Psi\left(x^{k}\right)$ for all $k$, and assume $x^{k} \rightarrow x$ and $z^{k} \rightarrow z$. Define $y^{k}$ by $y^{k}(t)=\dot{z}^{k}(t) / \lambda+z^{k}(t)$. Since $y^{k}(t) \in F_{t}\left(z^{k}(\cdot)\right)$ for almost all $t, y^{k}$ belongs to the unit ball in $L^{\infty}([0, T])$, which is equal to the dual of $L^{1}([0, T])$, for any $T \in[0, \infty)$. Therefore, by Alaoglu's theorem, a subsequence (again denoted by) $y^{k}$ converges in the weak* topology to some $y$ in $L^{\infty}([0, T])$, and hence, $y^{k}$ converges weakly to $y$ in $L^{1}([0, T])$ for any $T \in[0, \infty)$. Since $F_{t}(\cdot)$ is convex- and compact-valued upper semi-continuous correspondence (Lemma $5)$, we obtain $y(t) \in F_{t}(x(\cdot))$ almost everywhere due to the Convergence Theorem. 
On the other hand,

$$
z^{k}(t)=z^{k}(0)+\int_{0}^{t} \lambda\left(y^{k}(s)-z^{k}(s)\right) d s \rightarrow z(0)+\int_{0}^{t} \lambda(y(s)-z(s)) d s,
$$

i.e., $\dot{z}(t)=\lambda(y(t)-z(t))$ or $y(t)=\dot{z}(t) / \lambda+z(t)$ almost everywhere. Hence $\dot{z}(t) / \lambda+z(t) \in F_{t}(x(\cdot))$ almost everywhere, i.e., $z \in \Psi(x)$, which implies that the graph of $\Psi$ is closed. Since the values are contained in a compact set $K, \Psi$ is compact-valued and upper semi-continuous.

Now we are in a position to prove the equilibrium path existence theorem.

Proof of Theorem 1. Due to Kakutani's fixed point theorem (see, e.g., Aubin and Cellina [1, p. 85]), it follows from Lemma 6 that there exists $x^{*} \in K$ such that $x^{*} \in \Psi\left(x^{*}\right)$, which is a perfect foresight equilibrium path starting from an arbitrarily given $x^{0}$.

Remark 2. In order to establish the existence of perfect foresight equilibrium paths, it is sufficient to assume that $V[x](\cdot):[0, \infty) \rightarrow \mathbb{R}^{n}$ and $V[\cdot](t): K \rightarrow$ $\mathbb{R}^{n}$ are continuous.

\section{References}

[1] J.-P. Aubin and A. Cellina, "Differential Inclusions," Springer-Verlag, Berlin, 1984.

[2] K. Burdzy, D. M. Frankel, and A. Pauzner, Fast equilibrium selection by rational players living in a changing world, Econometrica 69 (2001), 163-189.

[3] H. Carlsson and E. van Damme, Global games and equilibrium selection, Econometrica 61 (1993), 989-1018.

[4] G. Ellison, Basins of attraction, long-run stochastic stability, and the speed of step-by-step evolution, Rev. Econ. Stud. 67 (2000), 17-45.

[5] D. Frankel, S. Morris, and A. Pauzner, "Equilibrium Selection in Global Games with Strategic Complementarities," mimeo, 2000.

[6] I. Gilboa and A. Matsui, Social stability and equilibrium, Econometrica 59 (1991), 859-867.

[7] J. C. Harsanyi and R. Selten, "A General Theory of Equilibrium Selection in Games," MIT Press, Cambridge, 1988.

[8] J. Hofbauer and G. Sorger, Perfect foresight and equilibrium selection in symmetric potential games, J. Econ. Theory 85 (1999), 1-23. 
[9] J. Hofbauer and G. Sorger, "A Differential Game Approach to Evolutionary Equilibrium Selection," mimeo, University of Vienna, 2000.

[10] A. Kajii and S. Morris, The robustness of equilibria to incomplete information, Econometrica 65 (1997), 1283-1309.

[11] M. Kandori, G. J. Mailath, and R. Rob, Learning, mutation, and long run equilibrium in games, Econometrica 61 (1993), 29-56.

[12] M. Kandori and R. Rob, Bandwagon effects and long run technology choice, Games Econ. Behav. 22 (1998), 30-60.

[13] Y. Kim, Equilibrium selection in n-person coordination games, Games Econ. Behav. 15 (1996), 203-227.

[14] T. Maruta, On the relationship between risk-dominance and stochastic stability, Games Econ. Behav. 19 (1997), 221-234.

[15] A. Matsui, Best response dynamics and socially stable strategies, $J$. Econ. Theory 57 (1992), 343-362.

[16] A. Matsui and K. Matsuyama, An approach to equilibrium selection, J. Econ. Theory 65 (1995), 415-443.

[17] S. Morris, R. Rob, and H. S. Shin, p-Dominance and belief potential, Econometrica 63 (1995), 145-157.

[18] J. Nash, Non-cooperative games, Ann. Math. 54 (1951), 286-295.

[19] T. Ui, Robust equilibria of potential games, Econometrica 69 (2001), 1373-1380.

[20] P. Young, The evolution of convention, Econometrica 61 (1993), 57-84. 